\title{
Rapid and efficient C-terminal labeling of nanobodies for DNA-PAINT
}

\author{
Valentin Fabricius*, Jonathan Lefèbre*, Hylkje Geertsema, Stephen F. Marino* and Helge Ewers* \\ Institute of Chemistry and Biochemistry, Freie Universität Berlin, 14195 Berlin, Germany \\ †E-mail: stmarino@gmx.de, helge.ewers@fu-berlin.de \\ *these authors contributed equally to this work
}

\begin{abstract}
Single molecule localization-based approaches to superresolution microscopy (SMLM) create images that resolve features smaller than the diffraction limit of light by rendering them from the sequentially measured positions of thousands of individual molecules. New SMLM approaches based on the transient binding of very bright dyes via DNA-DNA interaction (DNA-PAINT) allow the resolution of dyes only a few nanometers apart in vitro. This imaging of cellular structures requires the specific association of dyes to their targets, which results in an additional "linkage error". This error can be minimized by using extremely small, single-domain antibody-based binders such as nanobodies, but the DNA-oligomers used in DNA-PAINT are of significant size in comparison to nanobodies and may interfere with binding. We have here developed an optimized procedure based on enzymatic labeling and click-chemistry for the coupling of DNA oligomers to the nanobody C-terminus, which is located on the opposite side of the epitope-binding domain. Our approach allows for straightforward labeling, purification and DNAPAINT imaging. We performed high efficiency labeling of two different nanobodies and show dual color multiplexed SMLM to demonstrate the general applicability of our labeling scheme.
\end{abstract}




\section{Introduction}

Single molecule localization-based superresolution (SMLM) techniques such as PALM[1], STORM [2] and (d)STORM [3] are developing at a rapid pace [4-6] with significant improvements in optical strategies, image processing and analysis techniques. The precision with which a single fluorescent molecule can be localized depends largely on the number of photons detected and, with novel approaches involving bright dyes, molecules that are but $5 \mathrm{~nm}$ apart can be resolved [7]. While many aspects of SMLM have now reached a high level of maturity, one of the major challenges remains reducing the size of the fluorescent labels themselves to achieve resolutions on the order of the size of biological molecules. In SMLM, the target species itself is not directly imaged - rather, a fluorescent molecule that is either transiently or permanently associated with the target species is detected., with novel approaches, molecules that are spaced about $5 \mathrm{~nm}$ apart can be resolved [7]. While many aspects of SMLM have now reached a high level of maturity, one of the major challenges remains reducing the size of the fluorescent labels themselves to achieve resolutions on the order of the size of biological molecules. In SMLM, the target species itself is not directly imaged - rather, a fluorescent molecule that is either transiently or permanently associated with the target species is detected. The upper resolution limit of the experiment - the precision with which the target species can be localized - is therefore determined by the distance of the detected fluorophore from the center of the target species, referred to as 'linkage error'.

a

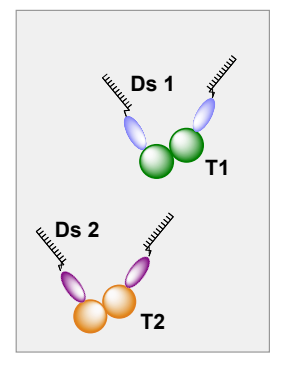

C

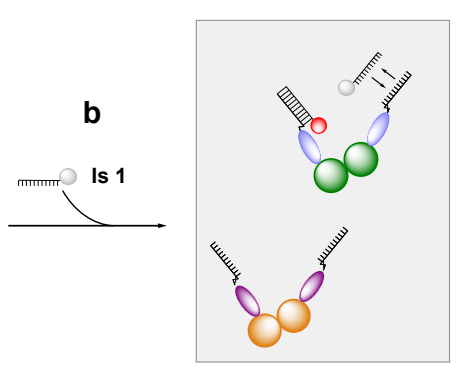

e

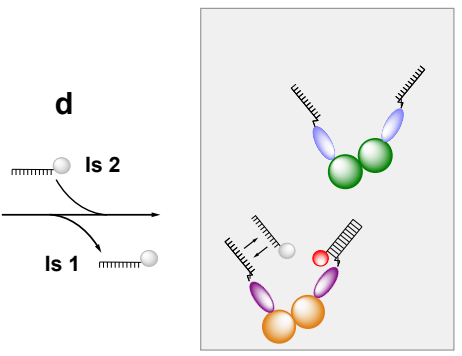

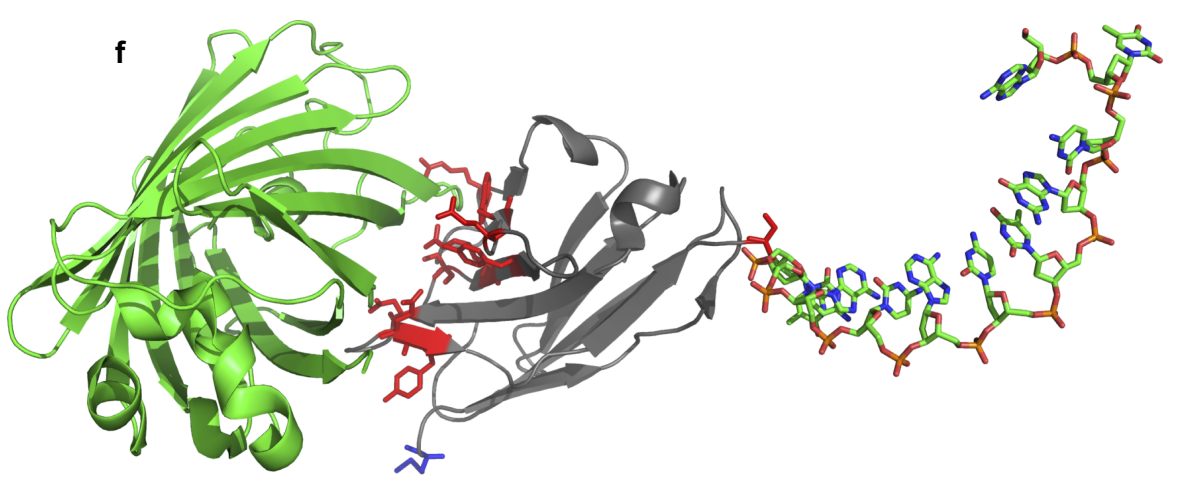

ca. $7.8 \mathrm{~nm}$

Figure 1. Schematic representation of multicolor DNA-PAINT with two docking-imager strand pairs (modified from [19]). (a) fixed cell stained with two different docking strand (Ds) carrying nanobodies bound to their respective targets. (b) Introduction of fluorophore carrying imager strand 1 (Is1). (c) Image acquisition of target 1. Docking and imager strand transiently interact, bringing the fluorophore into focus and allowing target localization (red). Unbound imager strands freely diffuse and are not localizable (grey). (d) Imager strand 1 is removed by washing and imager strand 2 (Is2) is introduced. (e) Image acquisition and localization of target 2. (f) Model of the docking strand labeled anti-GFP nanobody bound to its GFP target (modified from PDB accession code 3OGO); GFP is shown in green, the nanobody in grey, the nanobody GFP binding residues in red and the nanobody $\mathrm{N}$-terminus in blue. An oligonucleotide is attached $\mathrm{C}$-terminally to illustrate its relative size. 
The problem is readily appreciated when one considers an experiment that can theoretically achieve 40 $\mathrm{nm}$ resolution in the localization of a target that is detected via a fluorescently labelled IgG molecule placing the fluorophore up to $10 \mathrm{~nm}$ from the target's center (even further if the label is on a secondary antibody; consider also the error introduced due to the flexibility of such a fluorescent 'chain'). Clearly, the smaller the detected label, the better. Recently, small binders called nanobodies have been used to deliver dyes to within a few nm of the target structure [8] and indeed this improves resolution significantly in comparison to traditional immunolabeling [8]. Nanobodies are generalized single domain binding modules derived from the heavy-chain only immunoglobulins produced by camelids [9]. Their combination of an enormous potential binding repertoire, comparable and complementary to that of IgGs, in a robust domain obtainable via low cost and straightforward production has made them powerful tools for basic research and biotechnological applications [10-14]. Because these advantages come in a very small package $(\sim 15 \mathrm{kDa})$, nanobodies are quickly becoming indispensable for super resolution microscopic studies. The small size and compact structure of nanobodies (ca. 2-3 nm diameter, compared to ca. $10 \mathrm{~nm}$ for an $\mathrm{IgG}$ ) makes them very attractive as labels in super resolution experiments - particularly if fluorophores can be site-specifically attached via minimal length linkers. Their compact form also has a positive impact on the potential labeling density that can be achieved with them, particularly for continuous filamentous structures (eg. microtubules), minimizing the steric interference inherent to the use of much larger probes [15].

A recent improvement to both the ease and flexibility of labeling and detection techniques is provided by the 'DNA points accumulation for imaging in nanoscale topography' method (DNA-PAINT) (Figure 1), in which the target species, or a specific target detection module, is modified with a short DNA oligonucleotide (the "docking" strand) $[16,17]$. The position of the labeled species is then detected via addition of a complementary oligonucleotide harbouring a fluorophore (the "imager" strand). The low melting temperature $\left(\sim 25^{\circ} \mathrm{C}\right)$ of the short, corresponding duplex ensures a high imager strand $\mathrm{k}_{\text {off }}[17,18]$ and since the fluorophore can only be localized during the brief period the duplex exists, fluorophores are rapidly exchanged, thus constantly allowing new imager strands with fresh fluorophores to illuminate the target, thereby providing the necessary "blinking" for single-molecule localization microscopy (SMLM) (Figure 1). This technique allows the use of very bright, non-blinking dyes and antifade reagents, leading to significantly brighter localizations and thus higher resolution. DNA-PAINT using nanobodies has been reported for the anti-GFP nanobody, but the labeling was based on poorly selective succinimidylester chemistry combined with click-chemistry[19]. As shown in Figure 1f, the DNA-oligomer required for DNA-PAINT is of significant size in comparison with the nanobody and thus coupling directly to the $\mathrm{C}$-terminus as the site furthest away from the epitope-binding domain would be highly desirable. Here we report C-terminal labeling of anti-tubulin and anti-GFP nanobodies for DNA-PAINT. We have developed a streamlined procedure for the site-specific labeling of nanobodies based on the Sortase A (SrtA) reaction [20] and the copper-free strain promoted alkyneazide cycloaddition (SPAAC) [21] that improves both the ease of handling and the orthogonality of previously reported labeling schemes. The optimized procedure enables a great flexibility of label choice, allowing for multiplexed DNA-PAINT by coupling different oligo binder sequences to different nanobodies. Given their broad applicability we expect these contributions will substantially augment the current super resolution toolbox. 


\section{Methods}

\section{Expression and purification of nanobodies}

Expression constructs coding for the anti-tubulin nanobody [22] and anti-GFP nanobody [3,23] were previously described. The anti-tubulin nanobody construct was synthesized by GeneArt Gene Synthesis (Thermo Fisher Scientific) and the anti-GFP nanobody construct was obtained by PCR of pGEX-6P1containing anti-GFP-nanobody-4K [23]. PCR-amplified constructs were digested with NcoI and Bste 1 restriction enzymes and ligated in frame in similarly cut pHEN29 plasmid [15]. The pHEN29 plasmid comprises an $\mathrm{N}$-terminal pelB signal sequence, directing the nanobody to the periplasm, followed by a C-terminal Sortase A recognition sequence (LPETGG) upstream of a His 6 tag and an EPEA tag. Resulting constructs pHEN29-anti-GFP-LPETGG-His 6 -EPEA and pHEN29-anti-tubulin-LPETGGHis6-EPEA were confirmed by sequencing and transformed into E. coli WK6 for expression. Bacteria were grown in 11 of Terrific Broth medium (Carl Roth) supplemented with $1 \mathrm{mM} \mathrm{MgCl}_{2}, 0.1 \%$ Glucose, $0.4 \%$ glycerol and $100 \mu \mathrm{g} / \mathrm{ml}$ ampicillin at $37^{\circ} \mathrm{C}$, to an $\mathrm{OD}_{600}$ of $0.6-0.9$ in baffled shaking flasks. Expression was induced with $1 \mathrm{mM}$ Isopropyl- $\beta$-D-thiogalactopyranoside (IPTG), followed by incubation for $16-18 \mathrm{~h}$ at $28^{\circ} \mathrm{C}$. Cells were harvested by centrifugation $\left(10 \mathrm{~min}, 11,800 \mathrm{~g}, 4^{\circ} \mathrm{C}\right)$ and the resulting pellets were resuspended in $12 \mathrm{ml}$ ice-cold TES buffer $(0.2 \mathrm{M}$ Tris- $\mathrm{HCl}(\mathrm{pH} 8.0), 0.5 \mathrm{mM}$ EDTA, 0.5 M Sucrose) containing one protease inhibitor cocktail tablet (Roche) per $10 \mathrm{ml}$ of TES. After shaking for 1 hour at $4^{\circ} \mathrm{C}$, and $200 \mathrm{rpm}, 18 \mathrm{ml}$ ice-cold TES/4 (0.05 M Tris (pH 8.0), 0.125 mM EDTA, $0.125 \mathrm{M}$ Sucrose) were added and the cells were further incubated for 1 hour at $4^{\circ} \mathrm{C}$. After centrifugation $(11,800 \mathrm{~g}, 30 \mathrm{~min})$, the supernatant was collected, and the TES extraction repeated. The resulting periplasmic extracts were pooled and diluted 1:1 in wash buffer (20 mM HEPES (pH 7.5), $300 \mathrm{mM}$ $\mathrm{NaCl}, 5 \mathrm{mM}$ imidazole $10 \%$ glycerol) and applied to a $5 \mathrm{ml}$ HisPur cobalt column, pre-equilibrated in wash buffer at a flowrate of $3 \mathrm{ml}$ per minute using a peristaltic pump. The resin was washed with 10 bed volumes of wash buffer and His-tagged nanobodies were eluted with $20 \mathrm{mM}$ HEPES (pH 7.5), $300 \mathrm{mM}$ $\mathrm{NaCl}, 500 \mathrm{mM}$ imidazole and 10\% glycerol. Protein concentrations in eluted fractions were determined by absorbance at $280 \mathrm{~nm}$ using a NanoDrop1000 spectrophotometer (Thermo Fisher Scientific) using the calculated extinction coefficients. The nanobody was further purified and buffer exchanged by sizeexclusion chromatography over a Superdex 75 10/300 GL-column (GE Healthcare) in 20 mM HEPES (pH 7.5), $300 \mathrm{mM} \mathrm{NaCl}$ and 10\% glycerol on an ÄKTA Pure FPLC system. Nanobody-containing fractions were pooled, analyzed by SDS PAGE and concentrated using Amicon concentrators (3000 MWCO).

\section{Expression and purification of Sortase A}

Expression of the SrtA pentamutant (eSrtA, hereafter SrtA) in pET29 (kind gift from David Liu (Addgene plasmid \#75144)) was performed essentially as described with minor changes [24]. Briefly, E. coli BL21 DE3 were transformed with pET29-Sortase A pentamutant-His 6 and grown in 11 of LB medium, supplemented with $50 \mu \mathrm{g} / \mathrm{mL}$ kanamycin at $37^{\circ} \mathrm{C}$ to an $\mathrm{OD}_{600}$ of $\sim 0.5$. Expression was induced with $0.5 \mathrm{mM} \mathrm{IPTG}$ and the culture incubated at $30^{\circ} \mathrm{C}$ for 16 hours. Cells were harvested by centrifugation $\left(6000 \mathrm{x} \mathrm{g}, 4^{\circ} \mathrm{C}, 15 \mathrm{~min}\right)$ and resuspended in $50 \mathrm{ml}$ cold binding buffer $(20 \mathrm{mM}$ HEPES $\mathrm{pH} 7.5,150 \mathrm{mM} \mathrm{NaCl}$ ) and centrifuged again. Bacteria were lysed by resuspension in $25 \mathrm{ml}$ ice cold lysis buffer (50 mM HEPES pH 7.5, 300mM NaCl, $5 \mathrm{mM} \mathrm{MgCl}_{2}, 5 \mathrm{mM}$ Imidazole, 10\% Glycerol, $1 \mathrm{mg} / \mathrm{ml}$ DNase I, $1 \mathrm{mg} / \mathrm{ml}$ Lysozyme) and sonication on ice. The lysate was cleared by centrifugation 
(30 minutes, $16000 \times \mathrm{g}, 4^{\circ} \mathrm{C}$ ) and purified via HisPur cobalt and size-exclusion chromatography as for nanobody purification. SrtA containing fractions were pooled and concentrated with Amicon concentrators (3000 MWCO) to $25 \mathrm{mg} / \mathrm{ml}$ in $20 \mathrm{mM}$ HEPES pH 7.5, $300 \mathrm{mM} \mathrm{NaCl}, 10 \%$ Glycerol. The protein was analyzed by reducing SDS PAGE, snap-frozen in $100 \mu 1$ aliquots in liquid nitrogen and stored at $-80^{\circ} \mathrm{C}$ until further use.

\section{Sortase A-mediated coupling of clickable moieties (DBCO-amine)}

SrtA-mediated functionalization of sortagged nanobodies with Dibenzocyclooctyne- $\mathrm{NH}_{2}$ (DBCO-amine) (Sigma-Aldrich \# 761540) was carried out by reacting $50 \mu \mathrm{M}$ nanobody, $150 \mu \mathrm{M}$ SrtA and $10 \mathrm{mM}$ DBCO-amine (from a $50 \mathrm{mM}$ DMSO stock) in sortase buffer (20 mM HEPES (pH 7.5), $150 \mathrm{mM} \mathrm{NaCl}$, $10 \mathrm{mM} \mathrm{CaCl}_{2}$ ) for 1 hour at $24^{\circ} \mathrm{C}$. During the reaction, the GG-His6-EPEA peptide was cleaved from the nanobody to form a nanobody:SrtA covalent intermediate linked at the threonine in the LPET-sequence. Subsequent addition of DBCO-amine resolved the intermediate, substituting DBCO for SrtA at the LPET threonine residue. SrtA-His6-GG-His6-EPEA and unreacted His-tagged nanobody were separated from DBCO-nanobody conjugate using HisPur cobalt resin. For this, the reaction mixture was diluted 1:1 in $20 \mathrm{mM}$ HEPES (pH 7.5), $300 \mathrm{mM} \mathrm{NaCl}, 10 \%$ Glycerol, $5 \mathrm{mM}$ Imidazole, supplemented with 10 mM EDTA to stop the sortase reaction. Resin was washed with wash buffer until UV absorption at 280 $\mathrm{nm}$ reached baseline. Nanobody-DBCO containing flowthrough and wash fractions were pooled and remaining DBCO-amine was removed using an Amicon concentrator (3000 MWCO). NanobodyDBCO-conjugate concentrations were determined by Bradford-assay and successful conjugation and purity were assessed by SDS-PAGE. The degree of labeling (DOL) was determined by absorbance at $280 \mathrm{~nm}$ (nanobody) and $309 \mathrm{~nm}$ (DBCO) using a Nanodrop1000 spectrophotometer (Thermo Fisher Scientific) (Supplementary Table 2). The conjugate was stored at $4^{\circ} \mathrm{C}$ or immediately used for clickreactions. Optimal molar ratios of DBCO-amine:nanobody and reaction duration were determined in test reactions with $50 \mathrm{M}$ nanobody with $150 \mathrm{M}$ SrtA pentamutant in sortase buffer at $24^{\circ} \mathrm{C}$, with a DBCO-amine concentration between 2.5 and $12.5 \mathrm{mM}$ for $0.5-5$ hours.

\section{Copper-free SPAAC with azide-reaction partner}

Docking strand-oligonucleotides (Binder 1: TTATACATCTAG and Binder 3: TTTCTTCATTA), modified with a 5' azide moiety were synthesized by Microsynth (Switzerland) and dissolved in PBS (pH 7.4) (Sigma Aldrich) to a concentration of $10 \mathrm{mM}$. Alexa Fluor 647 (Invitrogen) containing an azide moiety was dissolved in DMSO to a final concentration of $10 \mathrm{mM}$. For a standard reaction, $40 \mu \mathrm{M}$ DBCO-nanobody conjugate was mixed with $40 \mu \mathrm{M}$ azide-reaction partner in $20 \mathrm{mM}$ HEPES ( $\mathrm{pH} 7.5$ ), $300 \mathrm{mM} \mathrm{NaCl}$ and $10 \%$ glycerol and incubated for $1 \mathrm{~h}$. Unbound reaction partners were removed in two buffer exchange steps with a Zeba spin desalting column (7000 MWCO). Successful labeling was verified by reducing SDS PAGE and analysis on a FUSION-FX7 Spectra fluorescence imager (Vilber Lourmat). The DOL was determined as for DBCO, using absorbance at $650 \mathrm{~nm}$ for the dye (Supplementary Table 2). Optimal molar ratios for the reaction were determined using azide-modified methoxy-polyethyleneglycol $\left(\mathrm{mPEG}, \mathrm{M}_{\mathrm{W}}=2000\right)$ as a click-reaction partner in molar ratios of nanobody to azide-PEG ranging from 1:1 to 1:10 and incubating for 0.5 to 12 hours. Reactions were quenched by adding $1 \mathrm{mM}$ DBCO-amine to the mixtures. Samples were analyzed on reducing SDS PAGE. 


\section{Cell culture and immunostaining}

HeLa cells, stably expressing CAV1-GFP (kind gift of the Helenius laboratory [25]) were maintained in DMEM (Life Tech) supplemented with $10 \%$ FCS and $1 \%$ Glutamax (Life Tech) at $37^{\circ} \mathrm{C}$ and $5 \% \mathrm{CO}_{2}$ in a humidified incubator. After 24 hours, cells were washed $3 \mathrm{x}$ with PBS and incubated in pre-extraction buffer (80 mM PIPES (pH 6.9), 1 mM MgCl 2,10 mM EGTA, $0.5 \%$ Triton X-100) for 60 seconds at room temperature. Pre-extraction buffer was replaced by fixation buffer ( $80 \mathrm{mM}$ PIPES (pH 6.9), $1 \mathrm{mM}$ $\mathrm{MgCl}_{2}, 1 \mathrm{mM}$ EGTA, $3.2 \%$ paraformaldehyde, $0.1 \%$ glutaraldehyde) and cells were incubated for 10 minutes at $37^{\circ} \mathrm{C}$. Fixation buffer was removed by washing with PBS and $10 \mathrm{mM}$ freshly prepared sodium borohydride in PBS was then added for 7 minutes followed by a ten-minute incubation in 100 $\mathrm{mM}$ glycine. Fixed cells were repeatedly washed with PBS and blocked with Image iTFX signal enhancer (Invitrogen) for 1 hour and then with $5 \%$ BSA and $0.1 \%$ Triton X-100 in PBS for 30 min at room temperature. After washing $3 \mathrm{x}$ with PBS, cells were stained with a $2.5 \mu \mathrm{g} / \mathrm{ml}$ solution of nanobodies, diluted in $5 \%$ BSA and $0.1 \%$ Triton X-100 in PBS. After 1 hour at room temperature, cells were washed $3 \mathrm{x}$ with PBS and immediately used for imaging.

\section{Optical setup}

Images were acquired with a Vutara 352 super resolution microscope (Bruker) equipped with a Hamamatsu ORCA Flash4.0 sCMOS for super resolution imaging and a 60x oil immersion TIRF objective with numerical aperture 1.49 (Olympus).

\section{Super-resolution dual color DNA PAINT imaging}

Imager strand oligonucleotides (Imager 1: CTAGATGTAT and Imager 3: GTAATGAAGA), modified with a 3' Atto 655 dye, were synthesized by Eurofins Genomics (Germany), aliqoted in PBS at a concentration of $100 \mu \mathrm{M}$ and stored at $-20{ }^{\circ} \mathrm{C}$ until imaging. For each sample, imager strand concentrations were empirically determined to ensure sufficiently high on-off ratios. Fixed samples were placed in a one-well magnetic chamber (Live Cell Instrument, South Korea) and covered in PBS (pH 7.4) supplemented with $0.5 \mathrm{M} \mathrm{NaCl}$ and $500 \mathrm{pM}$ Atto 655-labeled Imager 1. A $250 \mathrm{pM}$ solution of Atto 655 labeled Imager 3 was then added. Ideal exposure times for both binder-imager pairs were calculated with the Picasso Render tool [26]. Data were acquired with TIRF/HILO-illumination at a laser-power density of $2.5 \mathrm{~kW} / \mathrm{cm}^{2}$ using a $639 \mathrm{~nm}$ laser. Images were collected with a $100 \mathrm{~ms}$ acquisition time and typically 10000 images were used to reconstruct the super-resolution composites.

\section{Super-resolution data processing}

Raw data files were analyzed using the Picasso software package (https://github.com/jungmannlab/picasso). Individual binding events were identified by Picasso:Localize (Identification) with a box side length of 7 and a minimum net gradient of 1500 and 2000 for the microtubule and caveolae localizations, respectively. Subsequently, the localizations were loaded into Picasso:Render to correct for microscopic drift (Undrift by RCC), align microtubule and caveolin fluorescence and obtain binding kinetics. For our complementary oligos, we determined a $t_{\mathrm{on}}=600 \mathrm{~ms}$ and $t_{\text {off }}=118 \mathrm{~s}$ for binder-imager pair 1 and $t_{o n}=520 \mathrm{~ms}$ and $t_{o f f}=153 \mathrm{~s}$ for binder-imager pair 3 . Microtubule (250 nm straight sections without overlaps, aligned and processed as in [8]) and caveolin cross sections were taken in ImageJ and fitted to a Gaussian function to determine their respective full width at half maximum and interpeak distance. 
a

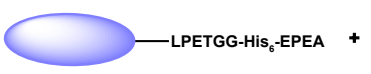

Nanobody $(\sim 15 \mathrm{kDa})$

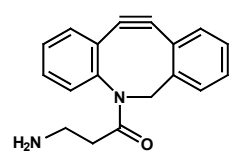

DBCO- $\mathrm{NH}_{2}(276 \mathrm{Da})$
Sortase A-mediated conjugation

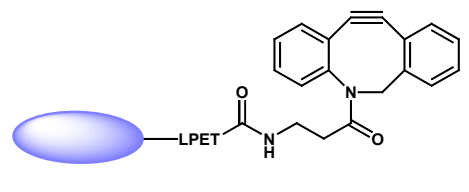

GG-His $_{6}$-EPEA

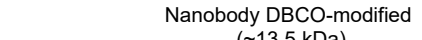

$(\sim 13.5 \mathrm{kDa})$

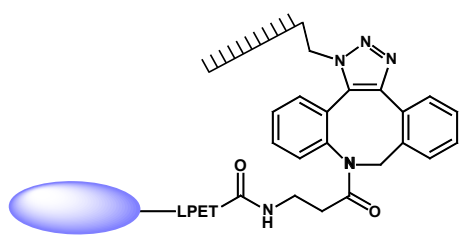

Nanobody-oligonucleotide conjugate $(\sim 17 \mathrm{kDa})$ Copper-free
click reaction click reaction

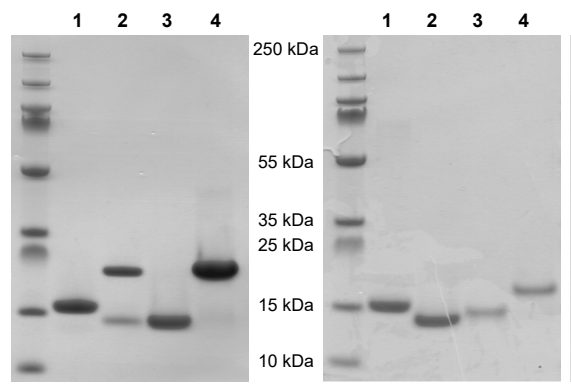
$(\sim 3.5 \mathrm{kDa})$

e

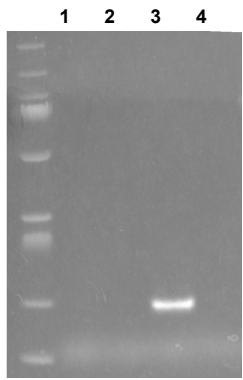

f

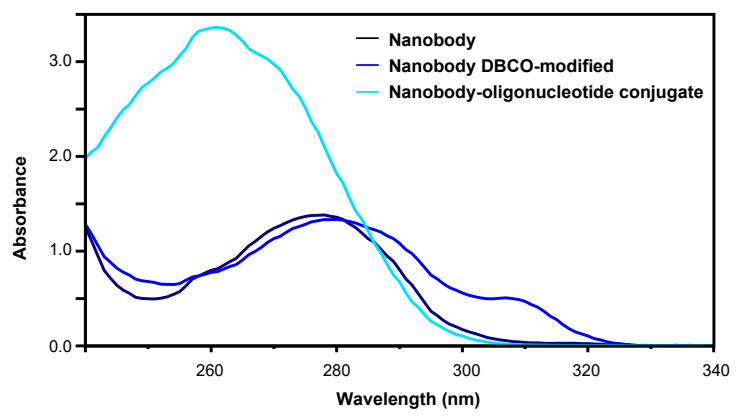

Figure 2. Schematic representation of the site-specific labeling of nanobodies via SrtA. (a) Srt A-mediated conjugation of DBCO amine to sortagged nanobody. (b) Copper-free click reaction of the nanobody-DBCO conjugate with azidemodified oligonucleotide. (c) Coomassie-stained SDS PAGE gel of samples taken during the SrtA-mediated conjugation of DBCO amine to anti-GFP nanobody. 1 = unmodified anti-GFP nanobody; 2 = reaction mixture after $1 \mathrm{~h}$ incubation; $3=$ pooled and concentrated DBCO-modified anti-GFP nanobody; 4 = elution of remaining His-tagged anti-GFP nanobody and SrtA. (d) Coomassie-stained SDS PAGE gel of samples taken before and after the click reaction. $1=$ unmodified anti-GFP nanobody; 2 = DBCO modified anti-GFP nanobody; 3 = Alexa Fluor 647 conjugated anti-GFP-nanobody; $4=$ docking strand 3 conjugated anti-GFP nanobody. (e) Fluorescence image of the SDS PAGE gel shown in (e), confirming fluorophore association. (f) UV absorbance spectra of the different conjugates. 


\section{Results}

\section{Nanobody production}

Recombinant anti-tubulin and anti-GFP-LPETGG-His 6 -EPEA nanobody constructs were produced in $E$. coli, extracted from the bacterial periplasm via osmotic shock and purified via immobilized metal ion and size exclusion chromatography to near homogeneity ( $>95 \%$ by SDS-PAGE). Typical final yields were between 15 and $20 \mathrm{mg} / \mathrm{l}$ culture for both constructs (Supplementary Figure 1). SrtA (ca. $40 \mathrm{mg} / 1$ culture) was similarly produced and purified.

\section{SrtA-mediated conjugation and copper-free SPAAC via DBCO-amine}

For derivatizing nanobodies with short oligonucleotides to use as labels in DNA-PAINT, we initially attempted a peptide exchange strategy using SrtA, similar to that of Massa et al. [15]. We used a SrtA pentamutant [24] to label two different probes: an anti-tubulin [22], and an anti-GFP nanobody [27]. Our substrate peptide carried a C-terminal alkyne moiety providing a specific reaction point for azide labeled 'docking' oligonucleotides using click chemistry via $\mathrm{Cu}(\mathrm{I})$ catalysed alkyne-azide cycloaddition (CuAAC) [28]. Although the reactions were successful (data not shown), we encountered several difficulties with the procedure (see Discussion), not least of which was the poor yield of final labeled nanobodies due to the need to remove $\mathrm{Cu}(\mathrm{I})$ from the reaction mix. In order to simplify the labeling procedure and maximize the yield, we sought to devise an efficient $\mathrm{Cu}(\mathrm{I})$ independent reaction scheme. $\mathrm{Cu}(\mathrm{I})$ free click reactions with biomolecules based on strain promoted alkyne-azide cycloaddition (SPAAC) using dibenzocyclooctyne (DBCO) have been reported and can be performed under physiological conditions [28-30]. Building on this idea, we tested whether an amine modified DBCO could directly function as the SrtA releasing nucleophile (Figure 2a). We again prepared SrtA-nanobody intermediates and subsequently added DBCO-amine in excess. We observed nearly quantitative conversion of the SrtA-nanobody intermediate and confirmed the association of one DBCO molecule per nanobody by absorption spectroscopy (Figure 2c, f; Supplementary Table 2) and Bradford assay. To confirm that the resulting constructs were competent for label addition, we optimized the labeling reaction using azide-modified $\mathrm{mPEG}\left(\mathrm{M}_{\mathrm{w}}=2000 \mathrm{Da}\right)$ at different concentrations and varying incubation times and monitored the results by electrophoretic mobility shift on SDS polyacrylamide gels. Nearly quantitative labeling was observed after 12 hours incubation with a 1:1 molar ratio of azide-modified reaction partner (Supplementary Figure 2). To assess whether the reaction yields conjugates with only one label per protein, Alexa-647-azide was reacted with nanobody:DBCO. Addition of Alexa-647-azide to the DBCO- nanobodies in a 1:1 ratio resulted in association of 1 fluorophore per nanobody, again confirmed by absorption spectroscopy (Figure 2d, e; Supplementary Table 2). The nanobodies performed well in conventional (d)STORM SMLM (not shown).

We then repeated our labeling reaction using azide-oligo docking strands. Again, we observed high efficiency addition of the oligos to the nanobodies based on electrophoretic mobility shift on SDS gels (Figure 2b, c).

\section{Dual color PAINT labeling}

The anti-tubulin- and anti-GFP-LPETGG-His6-EPEA nanobody constructs were coupled to previously reported oligo sequences binder 3 and binder 1, respectively [16]. The high efficiency of the SPAAC 
reaction of DBCO-functionalized nanobodies with the azide-modified docking strands allowed easy removal of unreacted oligonucleotides by simple buffer exchange.

We tested the viability of our nanobodies as super-resolution labeling probes by staining fixed Hela cells stably expressing CAV1-GFP with anti-tubulin nanobody coupled to binder 3 and anti-GFP nanobody coupled to binder 1. Sequential addition of dye-labeled imager 3 and imager 1 strands allowed us to acquire dual color DNA-PAINT images of cells (Figure 3). The microtubules were resolved with a full width half maximum of $31 \pm 4 \mathrm{~nm}$ and the plasma membrane caveolae with a diameter of $61 \pm 17 \mathrm{~nm}$ (Supplementary Figures 4, 5). These distances are far lower than the diffraction limit of light and agree well with previously reported microtubule [22] and caveolae diameters [31].
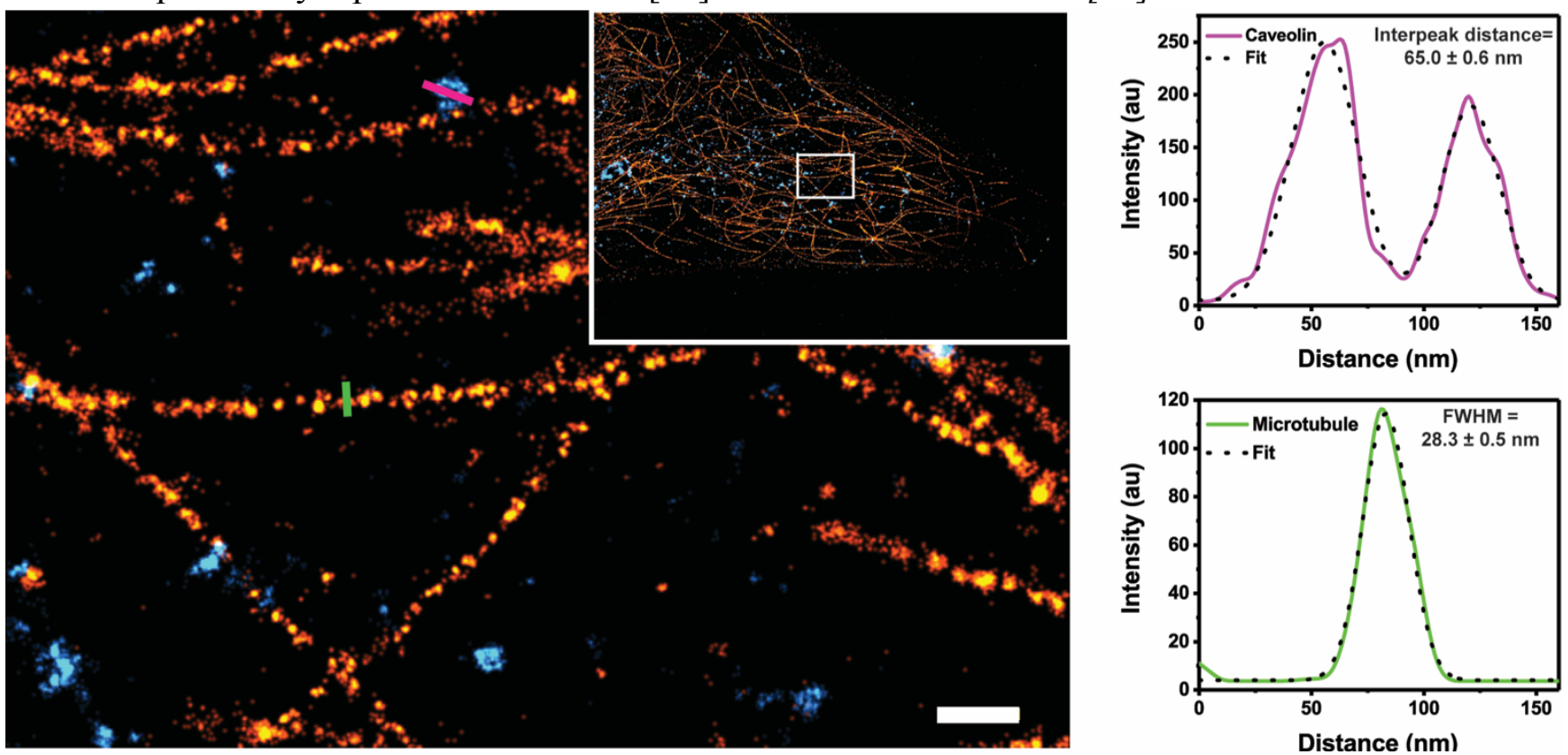

Figure 3. Dual color DNA-PAINT image of tubulin (orange) and caveolin (blue). On the left, resolved microtubules labeled with anti-tubulin-nanobody and caveolin-GFP labeled with anti-GFP-nanobody. Scale bar is $100 \mathrm{~nm}$. Inset, overview of the whole cell. The enlarged area is indicated with a white box. On the right, cross sections of a typical caveola (magenta) and microtuble (green) with full width at half maximum (FWHM) given for each, along with the fitting error. The NeNa localization precision [32] was $9 \mathrm{~nm}$ for both the microtubule and caveolin localizations. 


\section{Discussion}

The efficient conjugation of labels to an antigen-specific antibody or nanobody is a crucial first step for super resolution microscopy studies. Conventional protocols rely primarily on the use of $\mathrm{N}$ hydroxysuccinmide (NHS)- mediated conjugation of dyes to the $\varepsilon$-amine groups of lysine side chains. Since most proteins harbor several solvent-exposed lysines, this precludes site-specific label introduction and leads to non-uniform labeling of the probe, although specific labeling of the N-terminus has been reported [19]. Lysine residues can also occur near the antigen-binding site in the labeled protein, which can result in loss of affinity or complete inhibition of binding the target after dye conjugation.

Introducing an unpaired carboxy-terminal cysteine for conjugation via maleimide chemistry allows the site-specific introduction of a label without disturbing binding performance [33], but since IgGs and nanobodies require disulfide bonds for stability, the introduced cysteine can interfere with their proper folding. Even if this is not the case, the necessary reduction step prior to the labeling reaction can cause a substantial loss of material due to inadvertent reduction of the obligate disulfides [15]. Other sitespecific methods, including SrtA-mediated labelling, make use of chemoenzymatic reactions whose exquisite specificity guarantees the stability and homogeneity of labeling. This is especially desirable for DNA-PAINT labeling, as only stoichiometric labeling will make the probe compatible with quantitative approaches like qPAINT [34]. Since the oligonucleotide is quite large in comparison with the nanobody (see Figure 1f), its coupling close to the epitope-binding site in combination with its high charge may significantly perturb target recognition.

The SrtA variant from Staphyloccocus aureus recognizes an LPXTG-sequence (where X = any amino acid) on the target protein which it cleaves at the terminal glycine to form a target-SrtA acylintermediate at the preceding threonine, simultaneously releasing the C-terminal fragment. Subsequent nucleophilic attack by an N-terminal amine-containing species then releases SrtA, generating a hybrid target. The reaction is exploited for labeling by using probes containing a primary amine that can resolve the target-SrtA intermediate [35-37]. This reaction scheme allows specific coupling of a wide range of functional molecules to the C-terminus of any LPXTG-containing protein, including 'clickable' alkyne residues.

While CuAAC has been successfully applied for a variety of biomolecules, it requires a multi-step protocol and has the disadvantage of $\mathrm{Cu}(\mathrm{I})$ toxicity, thereby requiring removal of the metal for live cell experiments; additionally, $\mathrm{Cu}(\mathrm{I})$ can have a denaturing effect on proteins. Due to the need to remove $\mathrm{Cu}$ (I) from our reaction mixtures, we found it difficult to obtain satisfying yields of labeled nanobody. We therefore sought to eliminate $\mathrm{Cu}(\mathrm{I})$ altogether and reduce the number of steps in the labeling procedure by using SPAAC.

Van Lith et al. [38] used dibenzocyclooctyne (DBCO) in SPAAC as a general reactive moiety attached to a variable length PEG amine to provide a functionalizable linker for derivatization using various reaction chemistries. While this flexibility in linker length is intended to be beneficial for preparing antibody-drug conjugates (ADCs) for in vivo applications, it confers no advantage for super resolution fluorescence studies, where increasing linker length complicates the precise localization of the detected species. We therefore used DBCO-amine directly.

Baer et al., [39] in testing different amine nucleophiles for resolving the Srt A intermediate, observed a clear preference for small amine groups for the most efficient reactions. In our hands, resolution of the SrtA reaction using DBCO-amine was nearly quantitative. The specificity and low cost of the reagent 
provides the additional benefit that it can be used in large excess in the reaction to out-compete other potential nucleophiles (including the initially cleaved NH2-GGG-His6 peptide), thereby driving the desired reaction to completion.

By using DBCO-amine instead of $\mathrm{Cu}(\mathrm{I})$ in the reaction, we substantially reduced the preparation time of our samples while simultaneously increasing the efficiency of the labeling reaction and the overall yield of labeled probe. We suggest that the results here can be extended to accommodate the most diverse labeling strategies by utilizing small, bifunctional molecules having any compatible reactive group of interest coupled to an amine moiety that can complete the Sortase reaction. This approach facilitates the site-specific introduction of uniquely reactive tags into any peptide of interest, the hydrodynamic radius of whose subsequent reaction products need not exceed what the label itself adds. As the native Sortase reaction requires a small amine nucleophile (typically an N-terminal glycine for peptides), the high yields and simplified preparation of our nanobody conjugates achieved using DBCO-amine are likely to be mimicked using other small amine nucleophiles, greatly improving the ease and flexibility of preparing probes for super resolution studies. In our case, addition of the detection moiety to the Cterminus of the nanobodies ensures an accessible recognition sequence and that the modification has no detrimental effect on the binding interface, making the final constructs - always with a single label per molecule - ideal for a variety of quantitative studies (for example, single molecule brightness analyses, stepwise photobleaching, etc.). Taken together we here present a simple and efficient method for quantitative labeling of nanobodies for DNA-PAINT that due to the versatility of copper-free click chemistry provides an available labeling site at the very C-terminus of nanobodies for a variety of labeling schemes.

One of the great advantages of DNA-PAINT is the possibility of multiplexed labeling with the same dye on different epitopes where the specificity is encoded in the DNA-oligomers [16]. The combination with nanobodies holds great promise as more and more such binders are developed. In the future, highly multiplexed labeling will be possible using secondary nanobodies [40], nanobodies against peptide tags [41] and RFPs [14]. Further optimization of the labeling procedure will likely allow for unprecedented resolution using DNA-PAINT in cells.

\section{Acknowledgements}

We thank Jan Gettemans and Ralf Jungmann for advice and all members of the Ewers laboratory for helpful discussions. This work was supported by DFG through SFB958 INST 130/827-2 to H.E.

\section{References}

[1] Betzig E, Patterson G H, Sougrat R, Lindwasser O W, Olenych S, Bonifacino J S, Davidson M W, Lippincott-Schwartz J and Hess H F 2006 Imaging intracellular fluorescent proteins at nanometer resolution. Science 313 1642-5

[2] Rust M J, Bates M and Zhuang X 2006 Sub-diffraction-limit imaging by stochastic optical reconstruction microscopy (STORM). Nat Methods 3 793-5

[3] Heilemann M, van de Linde S, Schüttpelz M, Kasper R, Seefeldt B, Mukherjee A, Tinnefeld P and Sauer M 2008 Subdiffraction-resolution fluorescence imaging with conventional fluorescent probes. Angew. Chem. Int. Ed. Engl. 47 6172-6 
[4] Sauer M and Heilemann M 2017 Single-Molecule Localization Microscopy in Eukaryotes. Chem. Rev.

[5] Nicovich P R, Owen D M and Gaus K 2017 Turning single-molecule localization microscopy into a quantitative bioanalytical tool. Nat Protoc 12 453-60

[6] Hell S W, Sahl S J, Bates M, Zhuang X, Heintzmann R, Booth M J, Bewersdorf J, Shtengel G, Hess H, Tinnefeld P, Honigmann A, Jakobs S, Testa I, Cognet L, Lounis B, Ewers H, Davis S J, Eggeling C, Klenerman D, Willig K I, Vicidomini G, Castello M, Diaspro A and Cordes T 2015 The 2015 super-resolution microscopy roadmap J Phys D Appl Phys $\mathbf{4 8}$

[7] Dai M, Jungmann R and Yin P 2016 Optical imaging of individual biomolecules in densely packed clusters Nat Nanotechnol 11 798-807

[8] Ries J, Kaplan C, Platonova E, Eghlidi H and Ewers H 2012 A simple, versatile method for GFPbased super-resolution microscopy via nanobodies. Nat Methods 9 582-4

[9] Hamers-Casterman C, Atarhouch T, Muyldermans S, Robinson G, Hamers C, Songa E B, Bendahman N and Hamers R 1993 Naturally occurring antibodies devoid of light chains. Nature 363 446-8

[10] Beghein E and Gettemans J 2017 Nanobody Technology: A Versatile Toolkit for Microscopic Imaging, Protein-Protein Interaction Analysis, and Protein Function Exploration. Front Immunol 8771 [11] Schumacher D, Helma J, Schneider A F L, Leonhardt H and Hackenberger C 2017 Chemical functionalization strategies and intracellular applications of nanobodies. Angew. Chem. Int. Ed. Engl. [12] Muyldermans S, Baral T N, Retamozzo V C, De Baetselier P, De Genst E, Kinne J, Leonhardt H, Magez S, Nguyen V K, Revets H, Rothbauer U, Stijlemans B, Tillib S, Wernery U, Wyns L, Hassanzadeh-Ghassabeh G and Saerens D 2009 Camelid immunoglobulins and nanobody technology. Vet. Immunol. Immunopathol. 128 178-83

[13] Moutel S, Bery N, Bernard V, Keller L, Lemesre E, de Marco A, Ligat L, Rain J-C, Favre G, Olichon A and Perez F $2016 \mathrm{NaLi}-\mathrm{H} 1$ : A universal synthetic library of humanized nanobodies providing highly functional antibodies and intrabodies. Elife 5

[14] Fridy P C, Li Y, Keegan S, Thompson M K, Nudelman I, Scheid J F, Oeffinger M, Nussenzweig M C, Fenyö D, Chait B T and Rout M P 2014 A robust pipeline for rapid production of versatile nanobody repertoires. Nat Methods 11 1253-60

[15] Massa S, Vikani N, Betti C, Ballet S, Vanderhaegen S, Steyaert J, Descamps B, Vanhove C, Bunschoten A, van Leeuwen F W B, Hernot S, Caveliers V, Lahoutte T, Muyldermans S, Xavier C and Devoogdt N 2016 Sortase A-mediated site-specific labeling of camelid single-domain antibodyfragments: a versatile strategy for multiple molecular imaging modalities. Contrast Media Mol Imaging $11328-39$

[16] Jungmann R, Avendaño M S, Woehrstein J B, Dai M, Shih W M and Yin P 2014 Multiplexed 3D cellular super-resolution imaging with DNA-PAINT and Exchange-PAINT. Nat Methods

[17] Jungmann R, Steinhauer C, Scheible M, Kuzyk A, Tinnefeld P and Simmel F C 2010 Singlemolecule kinetics and super-resolution microscopy by fluorescence imaging of transient binding on DNA origami. Nano Lett 10 4756-61

[18] Mücksch J, Blumhardt P, Strauss M T, Petrov E P, Jungmann R and Schwille P 2018 Quantifying Reversible Surface Binding via Surface-Integrated Fluorescence Correlation Spectroscopy. Nano Lett 18 3185-92 
[19] Agasti S S, Wang Y, Schueder F, Sukumar A, Jungmann R and Yin P 2017 DNA-barcoded labeling probes for highly multiplexed Exchange-PAINT imaging. Chem Sci 8 3080-91

[20] Witte M D, Cragnolini J J, Dougan S K, Yoder N C, Popp M W and Ploegh H L 2012 Preparation of unnatural N-to-N and C-to-C protein fusions. Proc Natl Acad Sci USA 109 11993-8

[21] Agard N J, Prescher J A and Bertozzi C R 2004 A strain-promoted [3 + 2] azide-alkyne cycloaddition for covalent modification of biomolecules in living systems. J Am Chem Soc 126 15046-7

[22] Mikhaylova M, Cloin B M C, Finan K, van den Berg R, Teeuw J, Kijanka M M, Sokolowski M, Katrukha E A, Maidorn M, Opazo F, Moutel S, Vantard M, Perez F, van Bergen en Henegouwen P M P, Hoogenraad C C, Ewers H and Kapitein L C 2015 Resolving bundled microtubules using anti-tubulin nanobodies. Nat Commun 67933

[23] Platonova E, Winterflood C M, Junemann A, Albrecht D, Faix J and Ewers H 2015 Singlemolecule microscopy of molecules tagged with GFP or RFP derivatives in mammalian cells using nanobody binders. Methods

[24] Chen I, Dorr B M and Liu D R 2011 A general strategy for the evolution of bond-forming enzymes using yeast display. Proc Natl Acad Sci USA 108 11399-404

[25] Tagawa A, Mezzacasa A, Hayer A, Longatti A, Pelkmans L and Helenius A 2005 Assembly and trafficking of caveolar domains in the cell: caveolae as stable, cargo-triggered, vesicular transporters. $J$ Cell Biol 170 769-79

[26] Schnitzbauer J, Strauss M T, Schlichthaerle T, Schueder F and Jungmann R 2017 Superresolution microscopy with DNA-PAINT. Nat Protoc 12 1198-228

[27] Rothbauer U, Zolghadr K, Tillib S, Nowak D, Schermelleh L, Gahl A, Backmann N, Conrath K, Muyldermans S, Cardoso M C and Leonhardt H 2006 Targeting and tracing antigens in live cells with fluorescent nanobodies. Nat Methods 3 887-9

[28] Presolski S I, Hong V P and Finn M G 2011 Copper-Catalyzed Azide-Alkyne Click Chemistry for Bioconjugation. Curr Protoc Chem Biol 3 153-62

[29] Gong H, Holcomb I, Ooi A, Wang X, Majonis D, Unger M A and Ramakrishnan R 2016 Simple Method To Prepare Oligonucleotide-Conjugated Antibodies and Its Application in Multiplex Protein Detection in Single Cells. Bioconjug. Chem. 27 217-25

[30] Wagner K, Kwakkenbos M J, Claassen Y B, Maijoor K, Böhne M, van der Sluijs K F, Witte M D, van Zoelen D J, Cornelissen L A, Beaumont T, Bakker A Q, Ploegh H L and Spits H 2014 Bispecific antibody generated with sortase and click chemistry has broad antiinfluenza virus activity. Proc Natl Acad Sci USA 111 16820-5

[31] Platonova E, Winterflood C M and Ewers H 2015 A simple method for GFP- and RFP-based dual color single-molecule localization microscopy. ACS Chem. Biol. 10 1411-6

[32] Endesfelder U, Malkusch S, Fricke F and Heilemann M 2014 A simple method to estimate the average localization precision of a single-molecule localization microscopy experiment. Histochem Cell Biol

[33] Pleiner T, Bates M, Trakhanov S, Lee C-T, Schliep J E, Chug H, Böhning M, Stark H, Urlaub H and Görlich D 2015 Nanobodies: site-specific labeling for super-resolution imaging, rapid epitopemapping and native protein complex isolation. Elife $\mathbf{4}$

[34] Jungmann R, Avendaño M S, Dai M, Woehrstein J B, Agasti S S, Feiger Z, Rodal A and Yin P 2016 Quantitative super-resolution imaging with qPAINT. Nat Methods 
[35] Ritzefeld M 2014 Sortagging: a robust and efficient chemoenzymatic ligation strategy. Chemistry 20 8516-29

[36] Popp M W-L and Ploegh H L 2011 Making and breaking peptide bonds: protein engineering using sortase. Angew. Chem. Int. Ed. Engl. 50 5024-32

[37] Schmohl L and Schwarzer D 2014 Sortase-mediated ligations for the site-specific modification of proteins. Curr Opin Chem Biol 22 122-8

[38] van Lith S A M, van Duijnhoven S M J, Navis A C, Leenders W P J, Dolk E, Wennink J W H, van Nostrum C F and van Hest J C M 2017 Legomedicine-A Versatile Chemo-Enzymatic Approach for the Preparation of Targeted Dual-Labeled Llama Antibody-Nanoparticle Conjugates. Bioconjug. Chem. 28 539-48

[39] Baer S, Nigro J, Madej M P, Nisbet R M, Suryadinata R, Coia G, Hong L P T, Adams T E, Williams C C and Nuttall S D 2014 Comparison of alternative nucleophiles for Sortase A-mediated bioconjugation and application in neuronal cell labelling. Org. Biomol. Chem. 12 2675-85

[40] Pleiner T, Bates M and Görlich D 2017 A toolbox of anti-mouse and anti-rabbit IgG secondary nanobodies. J Cell Biol

[41] Braun M B, Traenkle B, Koch P A, Emele F, Weiss F, Poetz O, Stehle T and Rothbauer U 2016 Peptides in headlock--a novel high-affinity and versatile peptide-binding nanobody for proteomics and microscopy. Sci Rep 619211 
Supporting information for:

\section{C-terminal labeling of nanobodies for DNA-PAINT dual-color imaging}

Valentin Fabricius, Jonathan Lefèbre, Hylkje Geertsema, Stephen F. Marino and Helge Ewers

\section{Contents:}

Supplementary Table 1: Calculated molecular weight (MW) of nanobodies throughout the conjugation process.

Supplementary Table 2: Yield and degree of labelling (DOL) of SrtA-mediated labelling procedure and SPAAC reaction.

Supplementary Figure 1: Expression of anti-tubulin and anti-GFP nanobody-LPETGGHis $_{6}$-EPEA constructs.

Supplementary Figure 2: Analytical SDS-PAGE of mPEG-azide click reaction.

Supplementary Figure 3: Analytical SDS-PAGE of Alexa647-azide click reaction.

Supplementary Figure 4: Cross-sections of 7 different microtubule segments.

Supplementary Figure 5: Cross-sections of 7 different caveolae. 
Supplementary Table 1. Calculated molecular weight (MW) of nanobodies throughout the conjugation process.

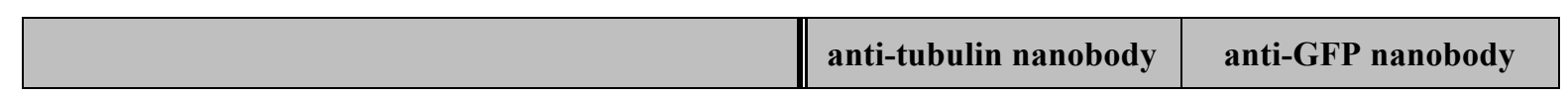

\begin{tabular}{|c|c|c|}
\hline MW of nanobody-LPETGG-His ${ }_{6}$ EPEA $^{1}$ & $15060 \mathrm{Da}$ & $14591 \mathrm{Da}$ \\
\hline MW of nanobody-LPET ${ }^{1}$ & $13697 \mathrm{Da}$ & $13227 \mathrm{Da}$ \\
\hline $\begin{array}{l}\text { MW of nanobody-LPET-DBCO } \\
\quad\left(\mathrm{MW}_{\mathrm{DBCO} \text { amine }} \approx 276 \mathrm{Da}\right)\end{array}$ & $13973 \mathrm{Da}$ & $13503 \mathrm{Da}$ \\
\hline $\begin{array}{l}\text { MW of nanobody-DBCO-mPEG } \\
\quad\left(\mathrm{MW}_{\mathrm{mPEG}} \approx 2000 \mathrm{Da}\right)\end{array}$ & $15973 \mathrm{Da}$ & $15503 \mathrm{Da}$ \\
\hline $\begin{array}{l}\text { MW of nanobody-DBCO-AlexaFluor } 647 \\
\qquad\left(\mathrm{MW}_{\mathrm{AF} 647} \approx 850 \mathrm{Da}\right)\end{array}$ & $14823 \mathrm{Da}$ & $14353 \mathrm{Da}$ \\
\hline $\begin{array}{l}\text { MW of nanobody-DBCO-docking strand oligo } \\
\qquad\left(\mathrm{MW}_{\text {oligo }} \approx 3700 \mathrm{Da}\right)\end{array}$ & $17673 \mathrm{Da}$ & $17203 \mathrm{Da}$ \\
\hline
\end{tabular}

${ }^{1}$ Calculated with ExPAsy ProtParam tool (expasy.org/protparam/) 
Supplementary Table 2: Yield and degree of labelling (DOL) of SrtA-mediated labelling procedure and SPAAC reaction. Values were calculated from measurements of three independent labeling reactions. Errors indicate the standard deviation of the averaged values.

anti-tubulin nanobody

anti-GFP nanobody

\begin{tabular}{|c||c|c|}
\hline $\begin{array}{c}\text { Total protein output/ input of the SrtA reaction x } \\
100 \%^{2}\end{array}$ & $90 \%$ & $93 \%$ \\
\hline $\begin{array}{c}\text { Average number of DBCO functionalities } \\
\text { incorporated per nanobody (DOL }\end{array}$ & $0.91 \pm 0.06$ & $0.90 \pm 0.04$ \\
\hline $\begin{array}{c}\text { Avero })^{3} \\
\text { Average number of AlexaFluor } 647 \text { azide labels per } \\
\text { nanobody }\left(\mathrm{DOL}_{\mathrm{AF} 647}\right)^{3}\end{array}$ & $0.94 \pm 0.03$ & $0.93 \pm 0.05$ \\
\hline
\end{tabular}

${ }^{2}$ Total protein concentration of nanobody used for the reaction (input) and nanobody after the SrtA-mediated conjugation and purification (output) were assessed with Bradford protein assay.

${ }^{3}$ Absorbance of the nanobodies $\left(\mathrm{A}_{280}\right)$ and the labelling probe $\left(\mathrm{A}_{\max }\right)$ were measured spectrophotometrically. DOL was calculated using Formula (1) with the calculated extinction coefficients of the respective nanobody $\left(\varepsilon_{\text {nanobody }}\right)$, of the labelling probe $\left(\varepsilon_{\max }\right)$ and the correction factor for the absorbance of the labelling probe at $280 \mathrm{~nm}(\mathrm{CF})$.

$$
D O L=\frac{c_{\text {labelling probe }}}{c_{\text {nanobody }}}=\frac{A_{\max } \varepsilon_{\text {nanobody }}}{\left(A_{280}-A_{\max } C F\right) \varepsilon_{\max }}
$$

With $\varepsilon_{\text {anti-GFP-LPET }}=27,055 \mathrm{M}^{-1} \mathrm{~cm}^{-1} ; \varepsilon_{\text {anti-tubulin-LPET }}=35,535 \mathrm{M}^{-1} \mathrm{~cm}^{-1} ; \varepsilon_{\max }(\mathrm{DBCO})=12,000 \mathrm{M}^{-1} \mathrm{~cm}^{-1} ; \varepsilon_{\max }$ $(\mathrm{AF} 647)=270,000 \mathrm{M}^{-1} \mathrm{~cm}^{-1} ; \mathrm{CF}_{\mathrm{DBCO}}=1.089 ; \mathrm{CF}_{\mathrm{AF} 647}=0.03 ; \lambda_{\max }(\mathrm{DBCO})=309 \mathrm{~nm} ; \lambda_{\max }(\mathrm{AF} 647)=650 \mathrm{~nm}$ 
a

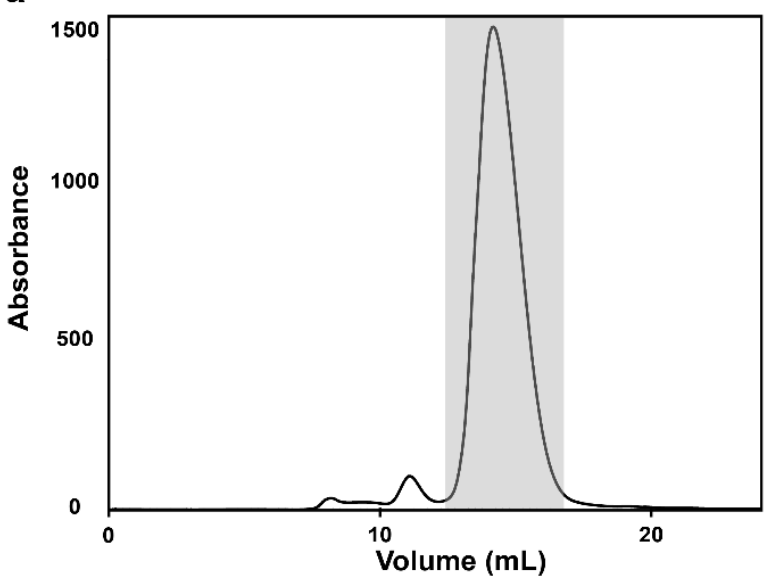

b

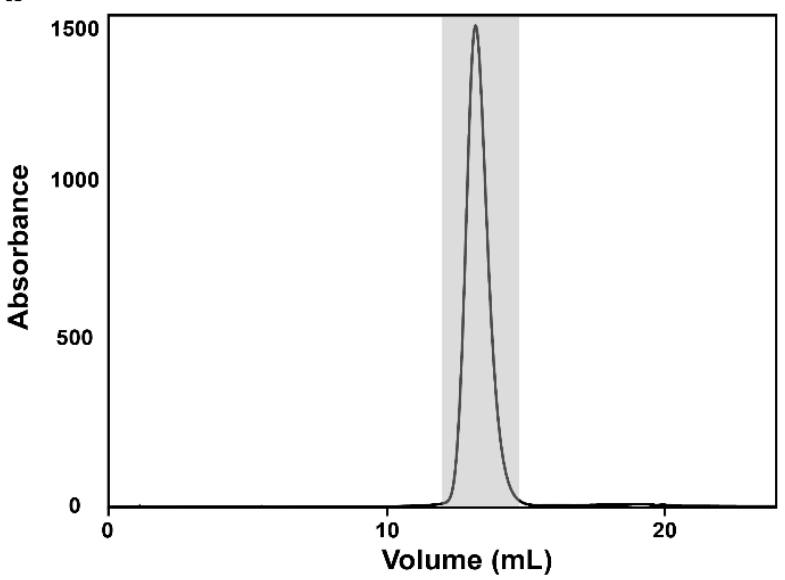

C

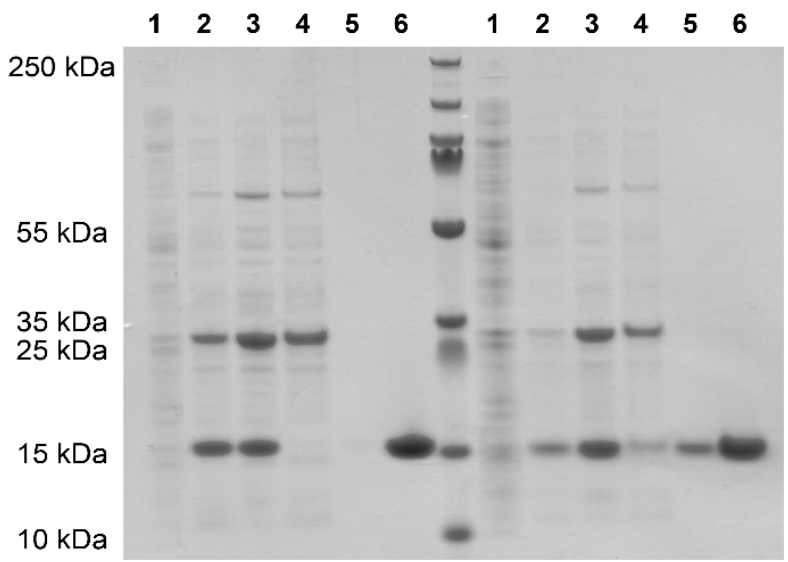

Supplementary Figure 1. Expression of anti-tubulin and anti-GFP nanobody-LPETGG-His 6 -EPEA constructs. (a) FPLC sizeexclusion chromatogram of anti-tubulin nanobody-LPETGG-His ${ }_{6}$-EPEA elution from HisPur resin. Nanobody-containing fractions are highlighted in grey. (b) FPLC chromatogram of anti-GFP nanobody-LPETGG-His 6 -EPEA elution from HisPur resin. Nanobody-containing fractions are highlighted in grey. (c) Coomassie-stained SDS PAGE gel of samples taken during the expression and purification of nanobodies. Lane $1=$ induced expression culture; lane $2=$ TES extract I; lane $3=$ TES extract II; lane $4=$ HisPur cobalt purification flow-through; lane $5=$ HisPur cobalt purification pre-elution; line $6=$ pooled nanobodycontaining fractions from the peaks highlighted in grey in (a) and (b).
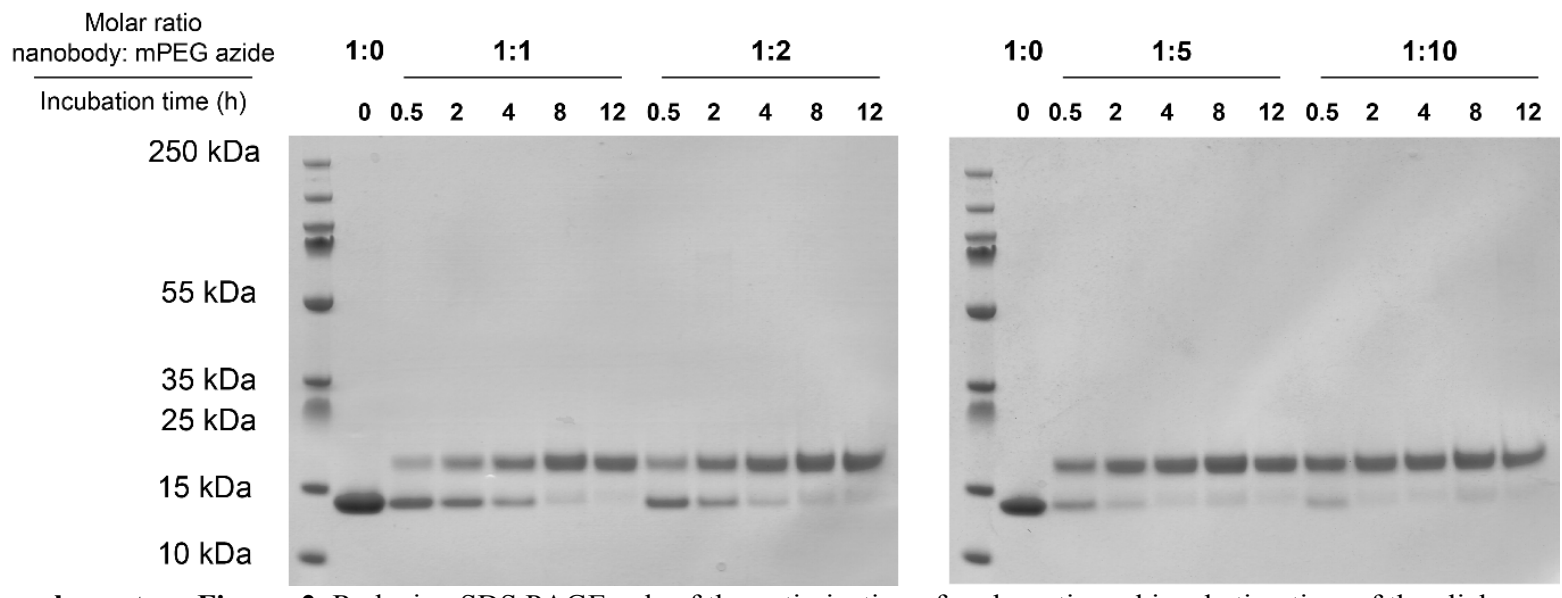

Supplementary Figure 2. Reducing SDS PAGE gels of the optimization of molar ratio and incubation time of the click reaction between nanobody-DBCO-conjugate and azide reaction partner, using $\mathrm{mPEG}$-azide (MW = $2000 \mathrm{Da})$ and anti-GFPDBCO nanobody conjugate. 
a

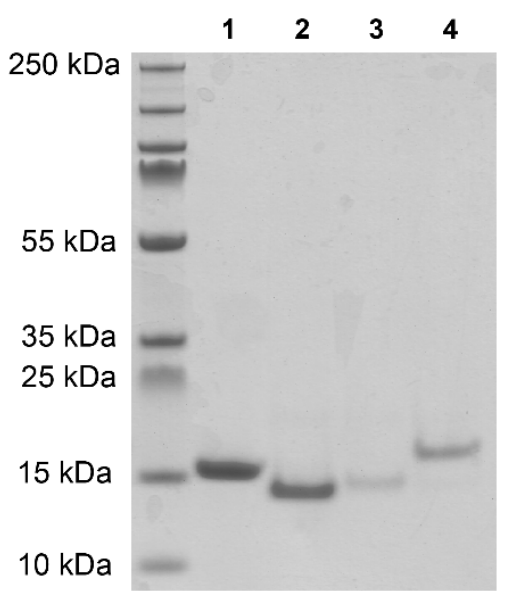

b

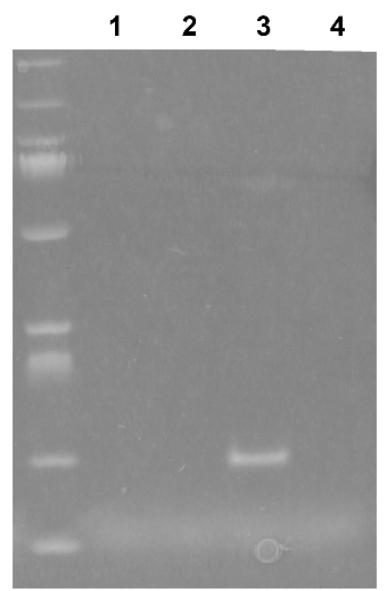

Supplementary Figure 3. Analytical SDS-PAGE of click reaction. (a) Coomassie-stained reducing SDS gel of samples taken before and after the click reaction. Lane $1=$ unmodified anti-tubulin nanobody; lane $2=$ DBCO modified anti-tubulin nanobody; lane 3 = Alexa Fluor 647 conjugated anti-tubulin-nanobody; lane 4 = docking strand 3 conjugated anti-tubulin nanobody. (b) Fluorescence image of the SDS PAGE gel shown in (e), confirming fluorophore association. 

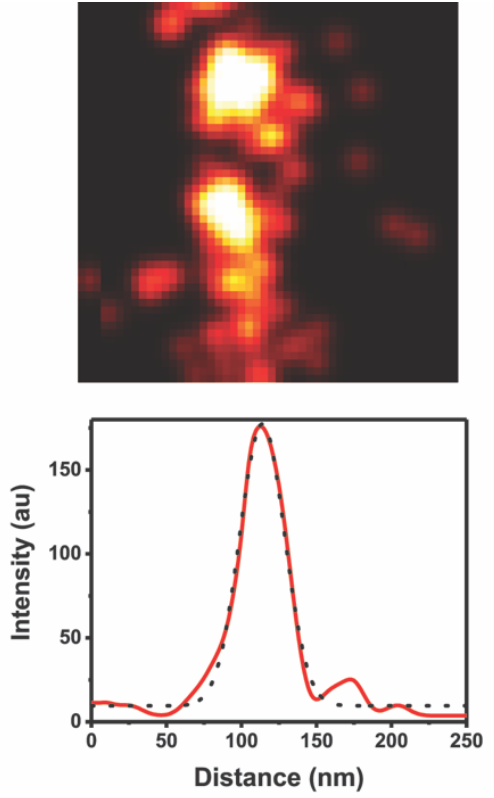

$F W H M=35.2 \pm 0.9 \mathrm{~nm}$
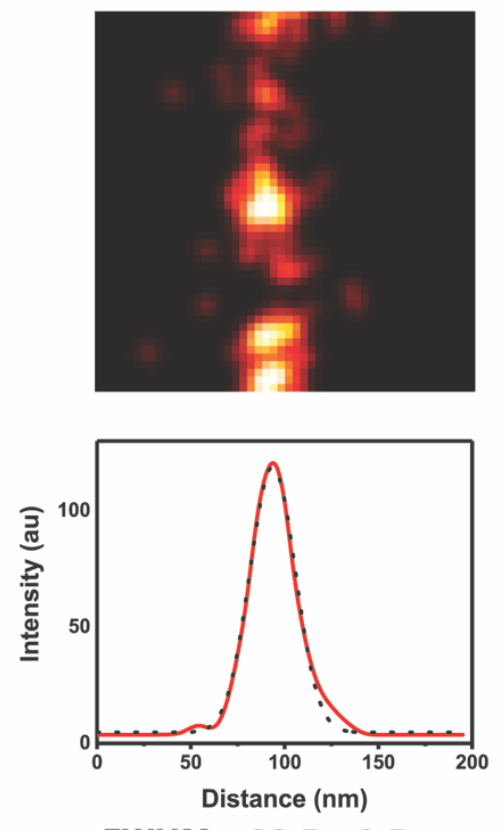

$F W H M=28.5 \pm 0.5 \mathrm{~nm}$
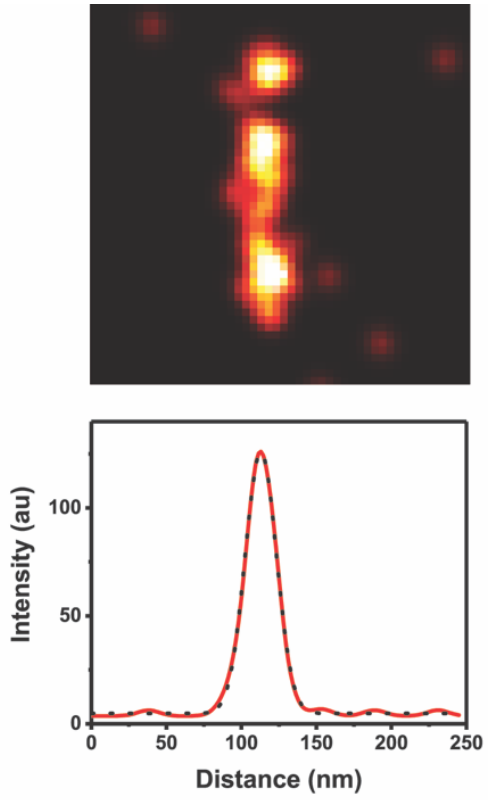

$F W H M=24.5 \pm 0.2 \mathrm{~nm}$
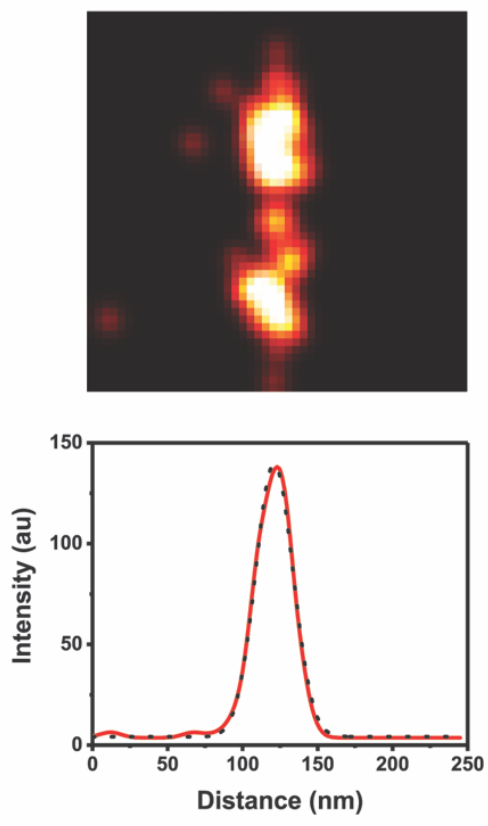

$F W H M=29.4 \pm 0.3 \mathrm{~nm}$
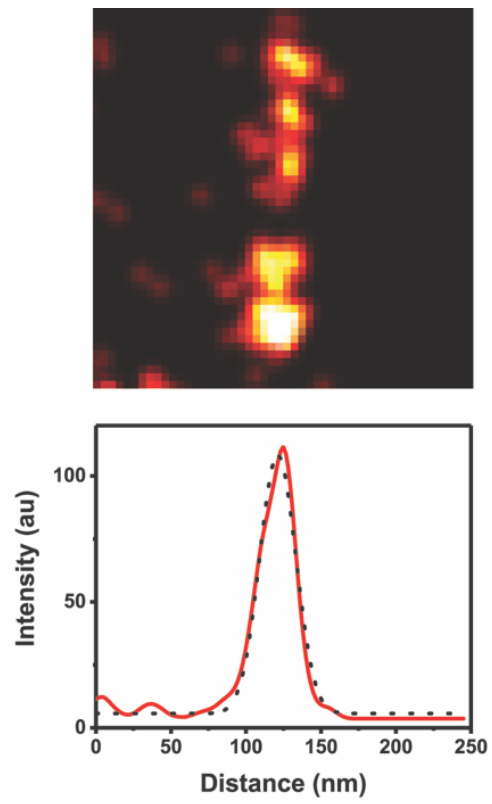

$F W H M=28.4 \pm 0.8 \mathrm{~nm}$
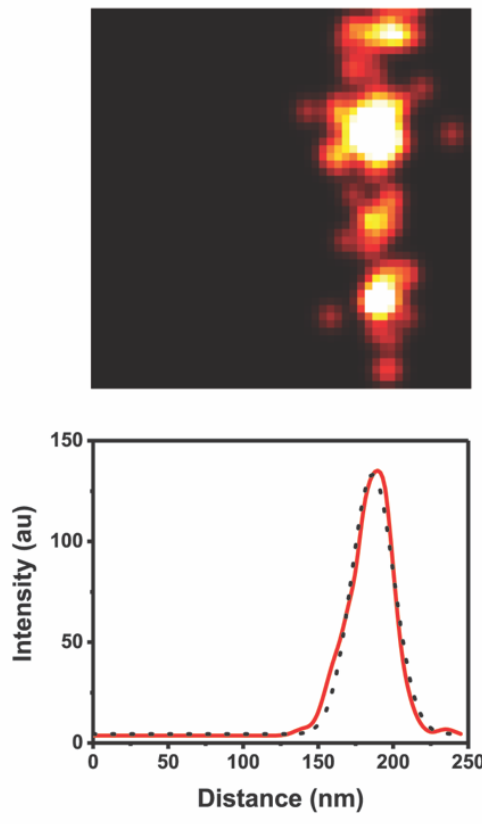

$F W H M=33.3 \pm 0.8 \mathrm{~nm}$
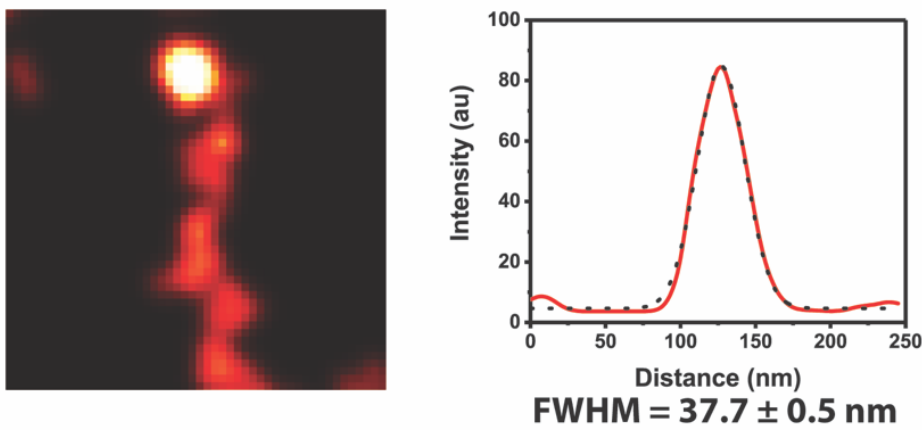

Supplementary Figure 4: Cross-sections of 7 different microtubule segments. Straight microtubule sections from 7 different areas $(250 \times 250 \mathrm{~nm})$ of the super-resolved images have been taken. Their cross-section is plotted and fitted with a Gaussian, from which the full-width half maximum (FWHM) was calculated. The error represents the fitting error. Taken together, the microtubules had a mean FWHM of $31 \pm 4$ (standard deviation) $\mathrm{nm}$. 

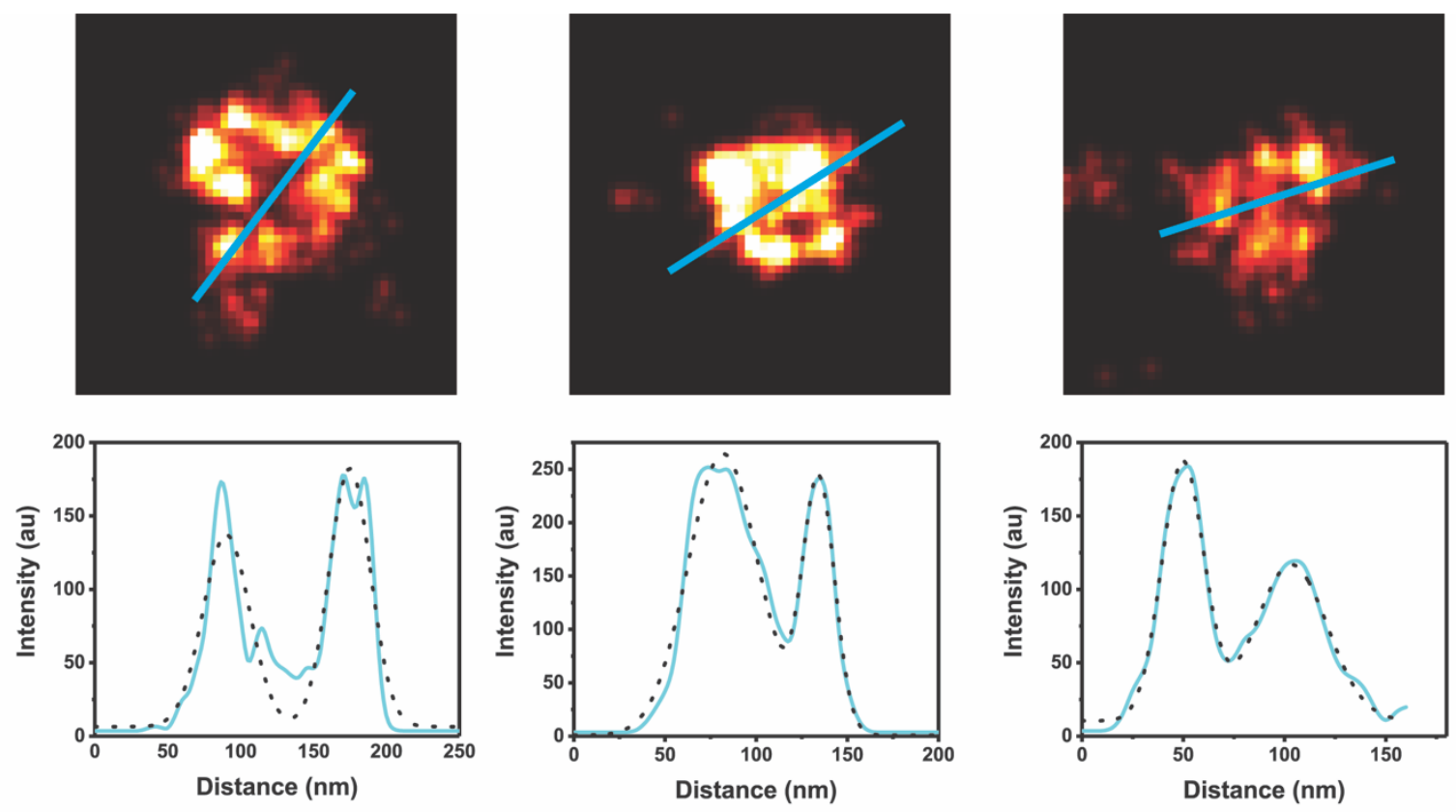

IPD $=84.7 \pm 1.1 \mathrm{~nm}$
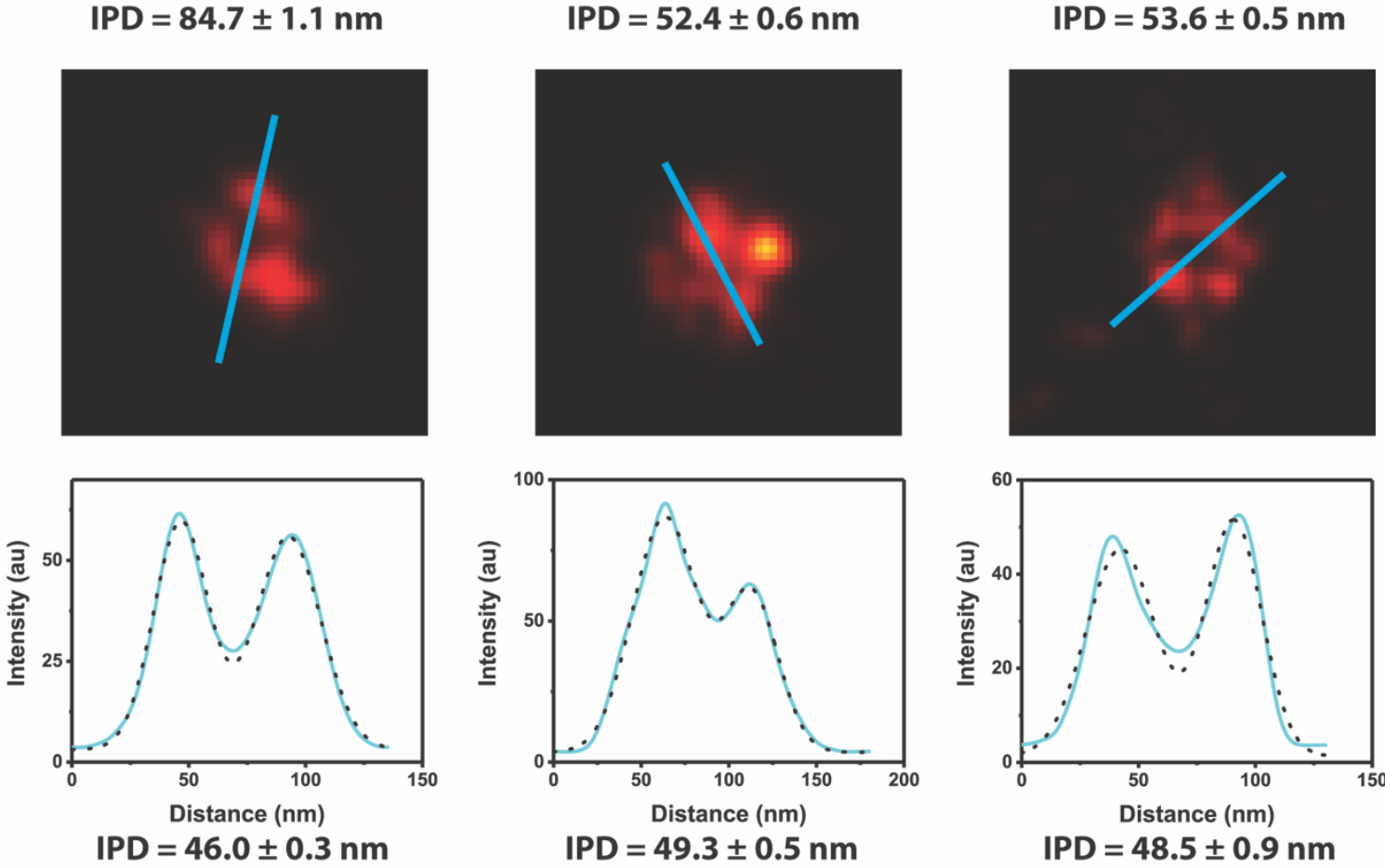

$$
\text { IPD }=49.3 \pm 0.5 \mathrm{~nm}
$$
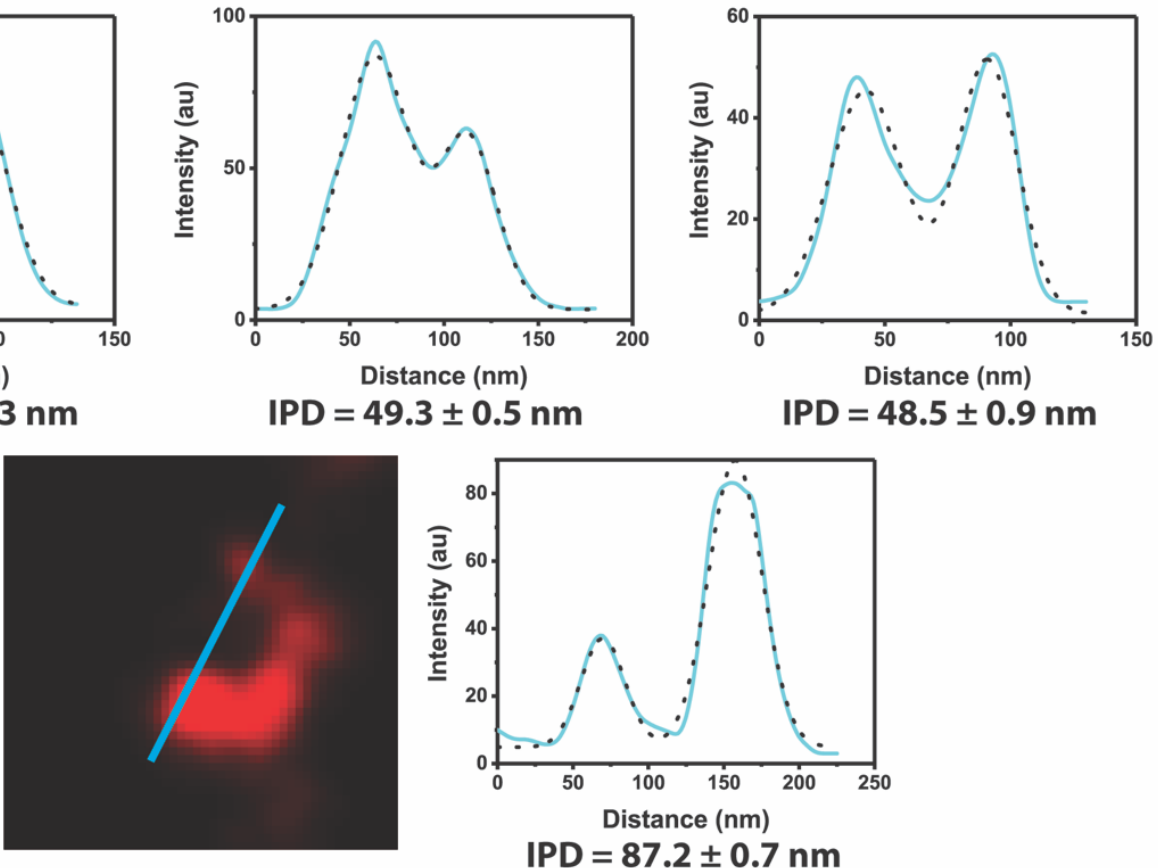

Supplementary Figure 5: Cross-sections of 7 different caveolae. From the super-resolved images, 7 different caveolae have been picked ( $250 \times 250 \mathrm{~nm}$ areas), their cross-section was plotted and fitted with a double Gaussian. The interpeak-distance (IPD) was calculated with the error representing the fitting error. Taken together, the caveolae had a mean diameter of $61 \pm$ 17 (standard deviation) $\mathrm{nm}$. 